\title{
Method for Analyzing E×B Probe Spectra from Hall Thruster Plumes
}

\author{
Rohit Shastry ${ }^{1}$, Richard R. Hofer ${ }^{2}$ \\ Jet Propulsion Laboratory, California Institute of Technology, Pasadena, CA 91109 \\ and \\ Bryan M. Reid ${ }^{3}$, Alec D. Gallimore ${ }^{4}$ \\ University of Michigan, Ann Arbor, MI 48109
}

\begin{abstract}
Various methods for accurately determining ion species' current fractions using $\mathbf{E} \times \mathbf{B}$ probes in Hall thruster plumes are investigated. The effects of peak broadening and charge exchange on the calculated values of current fractions are quantified in order to determine the importance of accounting for them in the analysis. It is shown that both peak broadening and charge exchange have a significant effect on the calculated current fractions over a variety of operating conditions, especially at operating pressures exceeding $10^{-5}$ torr. However, these effects can be accounted for using a simple approximation for the velocity distribution function and a one-dimensional charge exchange correction model. In order to keep plume attenuation from charge exchange below $30 \%$, it is recommended that $p z \leq 2$, where $p$ is the measured facility pressure in units of $10^{-5}$ torr, and $z$ is the distance from the thruster exit plane to the probe inlet. The spatial variation of the current fractions in the plume of a Hall thruster and the error induced from taking a single-point measurement are also briefly discussed.
\end{abstract}

\section{Nomenclature}

$\begin{array}{ll}Z & =\text { ion charge state } \\ e & =\text { electron charge } \\ d & =\text { ExB probe plate gap distance } \\ \vec{E} & =\text { electric field } \\ \vec{B} & =\text { magnetic field } \\ V_{\text {probe }} & =\text { ExB probe bias voltage } \\ u & =\text { ion velocity } \\ n & =\text { number density } \\ A_{c} & =\text { ExB probe collector area } \\ \Omega & =\text { ion current fraction } \\ \zeta & =\text { ion species fraction } \\ \vec{j} & =\text { ion current density } \\ m_{X e} & =\text { xenon atomic mass } \\ \sigma & =\text { charge-exchange cross section }\end{array}$

$\begin{array}{ll}\eta_{a} & =\text { anode efficiency } \\ \eta_{q} & =\text { charge utilization efficiency } \\ \eta_{v} & =\text { voltage utilization efficiency } \\ \eta_{d} & =\text { divergence utilization efficiency } \\ \eta_{b} & =\text { beam utilization efficiency } \\ \eta_{m} & =\text { mass utilization efficiency } \\ \theta & =\text { plume divergence half-angle } \\ V_{d} & =\text { thruster discharge voltage } \\ V_{a} & =\text { thruster acceleration voltage } \\ V_{l} & =\text { loss voltage } \\ I_{d} & =\text { thruster discharge current } \\ I_{b} & =\text { ion beam current } \\ \dot{m}_{a} & =\text { anode mass flow rate } \\ \alpha_{m} & =\text { mass utilization correction factor for }\end{array}$

\footnotetext{
${ }^{1} \mathrm{Ph}$. D. Candidate, Plasmadynamics and Electric Propulsion Laboratory, The University of Michigan, rshastry@umich.edu, and AIAA student member

${ }^{2}$ Technical Staff Member, Electric Propulsion Group, 4800 Oak Grove Dr., MS 125-109, Pasadena, CA 91109, richard.r.hofer@jpl.nasa.gov. AIAA senior member.

${ }^{3}$ Ph. D. Candidate, Plasmadynamics and Electric Propulsion Laboratory, reidb@umich.edu, and AIAA student member.

${ }^{4}$ Arthur F. Thurnau Professor of Aerospace Engineering and Director of the Plasmadynamics and Electric Propulsion Laboratory, alec.gallimore@umich.edu. AIAA Associate Fellow.
} 


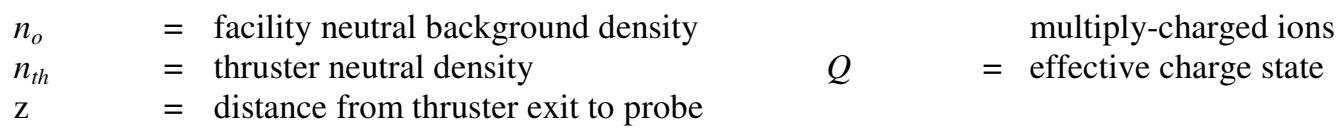

\section{Introduction}

$\mathrm{P}$ rformance and efficiency are important fundamental quantities in characterizing a Hall thruster and can require a large array of diagnostics to properly determine. While it has been shown that the majority of ions created in Hall thrusters are $\mathrm{Xe}^{+}[1-3]$, knowledge of the ion species' population is necessary to understand the competing processes affecting overall efficiency [4,5]. This population is typically measured using an E×B probe, which acts as an ion velocity filter. Since ion velocities in the plume are proportional to charge state, an $\mathrm{E} \times \mathrm{B}$ probe can differentiate between charge states, and species fractions can be determined from the amount of current the probe collects at each velocity. This diagnostic has been used successfully in the past on a variety of plasma sources $[1,2,4,6-8]$. However, analysis of probe spectra from Hall thrusters is not straightforward due to the broadening and blending of current peaks associated with each ion species. These features are caused by elastic collisions within the plume as well as a range of ion acceleration voltages within the channel, phenomena which are either less prominent or absent in ion thrusters. Furthermore, the high current densities associated with Hall thrusters typically results in higher facility operating pressures than ion thrusters. This results in larger amounts of charge exchange occurring within the plume, which affects $\mathrm{E} \times \mathrm{B}$ probe measurements typically performed far downstream of the thruster exit plane. Lastly, measurement of species population has relied on a single-point measurement done at thruster centerline, despite studies which show that this population varies within the plume [1,9]. All of these factors can result in an inaccurate determination of ion species' population in a Hall thruster plume.

The purpose of this study is to quantify the importance of including the above factors in determining current and species fractions in Hall thrusters. Various levels of correcting for species peak widths as well as charge exchange collisions were applied to $\mathrm{E} \times \mathrm{B}$ probe spectra from a 6-kW laboratory Hall thruster. The results from these methods were then compared to characterize the importance of correcting for the above factors. An analysis method is then recommended which is shown to provide the best balance between simplicity and accuracy.

The paper is organized as follows: Section II describes the experimental apparatus used to collect ExB spectra over a wide range of operating conditions. Section III illustrates the four methods studied to account for species peak width. Section IV details the model used to correct for charge exchange along with numerous simplifications and their validation. Section $\mathrm{V}$ gives the results of the comparison between analysis methods for several pertinent operating conditions. Section VI summarizes the results and provides recommendations based on them, discusses uncertainty generated by the additional analysis required in these methods, and addresses the issue of spatial variation of species fraction within the plume. Finally, Section VII gives the conclusions and recommendations of the study.

\section{Experimental Apparatus}

\section{A. ExB Probe}

An $\mathrm{E} \times \mathrm{B}$ probe, or Wien filter, is a band-pass ion filter that selects ions according to their velocities through the application of crossed electric and magnetic fields $[1,2,4,6-8,10]$. Most probes establish a constant magnetic field with permanent magnets while the electric field is established between two parallel plates. Sweeping the plate voltage while monitoring the ion current that passes through the probe yields a currentvoltage characteristic that is related to the ion velocity distribution function. Because the velocity of multiplycharged ions in Hall thrusters is proportional to the square root of their charge state, an $\mathrm{E} \times \mathrm{B}$ probe can be used to discriminate between ion species. Analysis of the ion current from the probe characteristic can then be

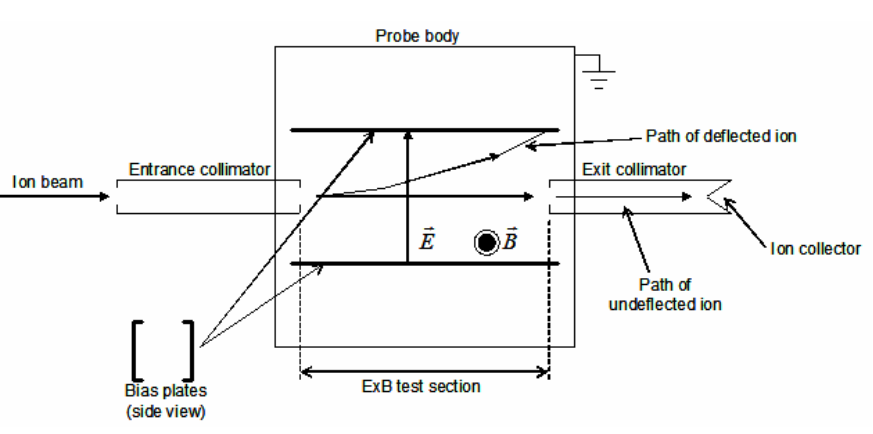

Figure 1: Schematic of ExB probe. Note: Not to scale. 
used to compute the ion species fractions.

As shown in Figure 1, the $\mathrm{E} \times \mathrm{B}$ probe is made up of three main sections: the entrance collimator, $\mathrm{E} \times \mathrm{B}$ test section, and exit collimator. Ions passing through the entrance collimator must travel through the test section undeflected to reach the collector. The motion of an ion through the test section is described by the Lorentz force equation given by

$$
\vec{F}=e Z(\vec{E}+\vec{u} \times \vec{B}) .
$$

The test section filters particles with a particular velocity by balancing the electric and magnetic fields such that there is no net force acting on those particles. Permanent magnets are usually employed to establish a constant magnetic field while the electric field is typically established between two parallel plates separated by a gap distance $d$ and biased to a potential $V_{\text {probe }}$. Setting the force equal to zero in Equation 1, the velocity of an ion passing through the test section undeflected is

$$
u_{\text {pass }}=\frac{E}{B}=\frac{V_{\text {probe }}}{B d} .
$$

Since the gap distance and magnetic field are fixed, the ion velocity is proportional to the probe voltage. Thus, the probe voltage can be swept across an appropriate range to capture the current from various charge states. The current collected at any given voltage can be written as:

$$
I_{i}=e Z_{i} n_{i} u_{i} A_{c}=e Z_{i} n_{i} \sqrt{\frac{2 e Z_{i} V_{a, i}}{m_{X e}}} A_{c},
$$

where $Z_{i}$ is the ion's charge state, $n_{i}$ is the number density, $V_{a, i}$ is the ion's acceleration voltage, and $A_{c}$ is the probe collection area. The second term assumes the ions were accelerated electrostatically through potential $V_{a, i .}$. Secondary electron emission effects are not included in this particular analysis as in Ref. [4] because of the use of a specially shaped collector that recollects any secondary emission current. Once currents from each species are measured, they can be used to determine their respective current fractions defined as

$$
\Omega_{i}=\frac{I_{i}}{\sum I_{i}}=\frac{n_{i} Z_{i}^{3 / 2}}{\sum n_{i} Z_{i}^{3 / 2}} .
$$

The second term neglects the variation in acceleration voltage across species as they tend to only differ by a few tens of volts [4]. Noting that the denominator in Equation 4 is a normalization factor, it can be inverted to determine the corresponding species fractions given by

$$
\zeta_{i}=\frac{n_{i}}{\sum n_{i}}=\frac{\Omega_{i} / Z_{i}^{3 / 2}}{\sum^{\Omega_{i}} / Z_{i}^{3 / 2}} .
$$

The E $\times \mathrm{B}$ probe used in these experiments was used previously during the NSTAR extended life test at the Jet Propulsion Laboratory (JPL) [11]. The probe was positioned $1.9 \mathrm{~m}$ downstream of the thruster exit plane on thruster centerline. The entrance collimator was $13.4 \mathrm{~cm}$ in length and had two circular orifices at either end that were 0.027 $\mathrm{cm}$ in diameter. In the $12.7-\mathrm{cm}$-long test section, the magnetic field was applied with permanent magnets that provided a magnetic field strength at the test section center of $0.1 \mathrm{~T}$. The electric field was established with a pair of aluminum plates machined from channel stock. The bias plates were separated by a distance of $d=1.9 \mathrm{~cm}$ with legs used to minimize electric field fringing that were $d / 4$ in length [10]. The exit collimator was $4 \mathrm{~cm}$ long and had an entrance orifice diameter of $0.027 \mathrm{~cm}$. A concave-shaped, tungsten collection electrode was placed at the end of the 
exit collimator. The concave shape was chosen so that secondary electrons emitted from the collector would be recollected. The acceptance angle of the probe was less than $0.1^{\circ}$.

\section{B. Faraday Probe}

Surveys of the ion current density in the thruster plume were taken using a Faraday probe. The probe consisted of a 1.9-cm-diameter collection electrode enclosed within a $2.5-\mathrm{cm}$-diameter guard ring. The guard ring and collector were separated by a $0.1-\mathrm{cm}$ gap, were fabricated from graphite, and were biased $-30 \mathrm{~V}$ below facility ground to repel electrons.

\section{Vacuum Facility}

Experiments were performed in the Endurance Test Facility (ETF) at JPL. The 3-m-diameter by 10-m-long vacuum chamber was previously used for the $30 \mathrm{kh}$ life test of the $2.3 \mathrm{~kW}$ NSTAR ion thruster and has also been used to test the NEXIS ion thruster at power levels exceeding $20 \mathrm{~kW}[11,12]$. The facility is cryogenically pumped and is lined with graphite panels to minimize backsputtered material to thruster surfaces. Base pressures between $10^{-8}$ and $10^{-7}$ torr are routinely achieved. At a total xenon flow rate of $22.5 \mathrm{mg} / \mathrm{s}$ the operating pressure was $1.6 \times$ $10^{-5}$ torr.

\section{Hall Thruster}

Experiments were performed using a 6-kW laboratory model Hall thruster that has an approximate throttling range of 100-500 $\mathrm{mN}$ thrust and 1000-3000 s specific impulse. The hollow cathode used to maintain and neutralize the discharge was mounted on the thruster centerline inside the inner magnetic core of the thruster. The cathode was always operated at $7 \%$ of the anode mass flow rate. Power and propellant were delivered to the thruster with commercially available power supplies and flow controllers. The plasma discharge was sustained by a matching pair of power supplies wired in parallel that provided a maximum output of $500 \mathrm{~V}, 40 \mathrm{~A}$. The discharge filter consisted of a $40 \mu \mathrm{F}$ capacitor in parallel with the discharge power supply outputs. Additional power supplies were used to power the magnet coils and the cathode heater and keeper. The cathode heater and keeper were used only during the thruster ignition sequence. Research-grade xenon (99.9995\% pure) was supplied through stainless steel feed lines with 50 and $500 \mathrm{sccm}$ mass flow controllers. The controllers were calibrated after the experiment and were digitally controlled with an accuracy of $\pm 1 \%$ of the set point.

\section{Methods for Determining Current Fractions}

As mentioned in the previous section, current fractions for each species must be determined from the ExB probe spectrum in order to quantify their relative populations. Determination of these fractions, however, is not straightforward due to the broadening and blending of peaks associated with each charge state. This effect is caused by a variety of factors, such as the presence of a range of acceleration voltages within the thruster (as all ions are not created in the same location) as well as collisional effects within the plume. These features are more prominent in spectra from Hall thruster plumes; spectra from ion engines contain flatter, more well-defined peaks due to their clear separation of ionization and acceleration zones, making data analysis more straightforward. The analysis of the resulting velocity distribution functions (VDF) was performed rigorously by Kim [1]. However, the present study is not concerned with such detailed analyses of the measured VDFs, but rather in quantifying the importance of the VDF in calculating current and species fractions with minimal uncertainty. Motivation for including the entire peak within this calculation was suggested by Beal [13]. If all species are subject to the same range of acceleration voltages, then it can be shown from the electrostatic acceleration and $\mathrm{E} \times \mathrm{B}$ probe equations that:

$$
\Delta V_{\text {probe }} \sim \Delta u \sim Z_{i}^{1 / 2} .
$$

This indicates that the range in probe voltages induced by the range in acceleration voltages is naturally larger for higher charge states. Thus, simply neglecting this broadening by only using the peak heights to characterize each species [4] may introduce higher uncertainty into the calculated current fractions.

In order to determine the importance of including the peak width in the calculation of current fractions, four different analysis methods were employed and compared: peak heights, triangle fitting, Gaussian fitting, and variable exponential fitting. A description of each of these methods can be found below. 


\section{A. Method of Peak Heights}

This method, suggested by Hofer [4], is the simplest and most straightforward of the four investigated. Under the assumption that the variation in peak widths can be neglected, the current for each species is taken as the maximum of its corresponding peak (see Figure 2). While this method largely ignores peak width and the overlap areas between peaks, it is attractive due to its simplicity and ease of automation.

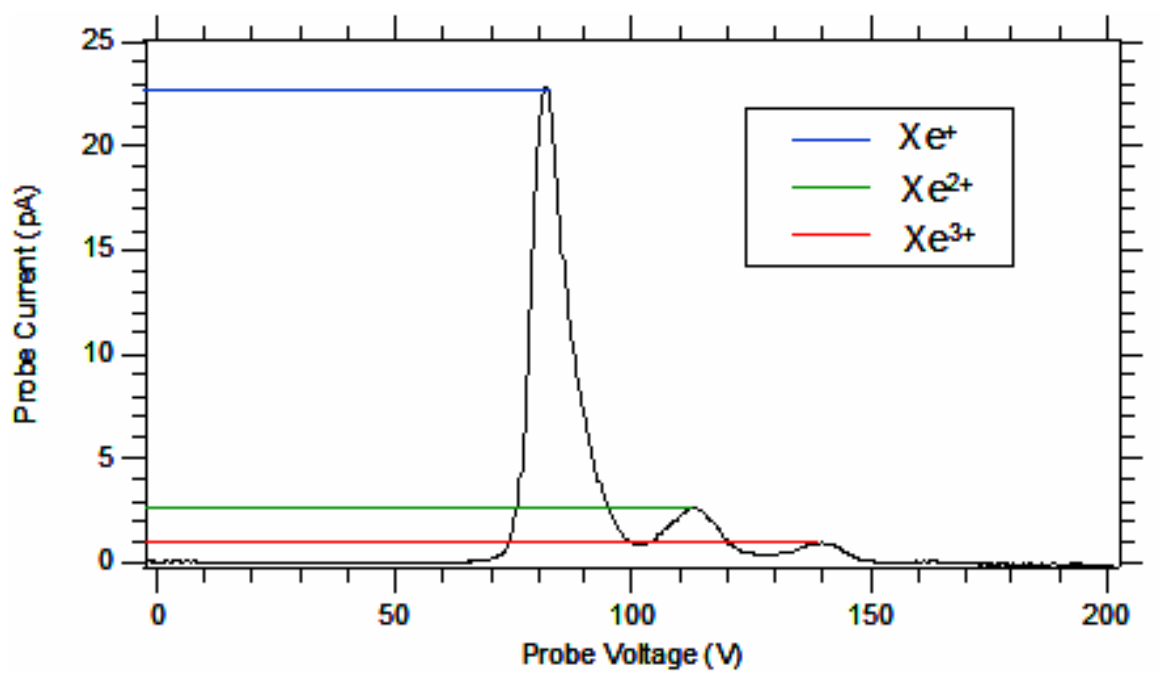

Figure 2: Illustration of the method of peak heights.

\section{B. Method of Triangle Fitting}

This method, suggested by Beal [13], is a simple, first-order method to include the effects of peak broadening in current fraction determination. Triangles are effectively drawn over each peak using lines which connect the peak height and the point of half-maximum. Since the right side of each peak is typically more well-defined than the left, this line is drawn on the right side of each peak and mirrored on the left to create a symmetric triangle (see Figure 3 ). The area of this triangle is then taken as the current collected for the corresponding species. It can be shown that the product of the maximum current and the half-width at half-maximum (HWHM) is proportional to the area of the full triangle. Since these values will only be used to calculate ratios, this product is used as a measure of the collected current. While this method is only a rough measure of the total current collected for each peak, it captures a large amount of the broadening effect while remaining relatively straightforward.

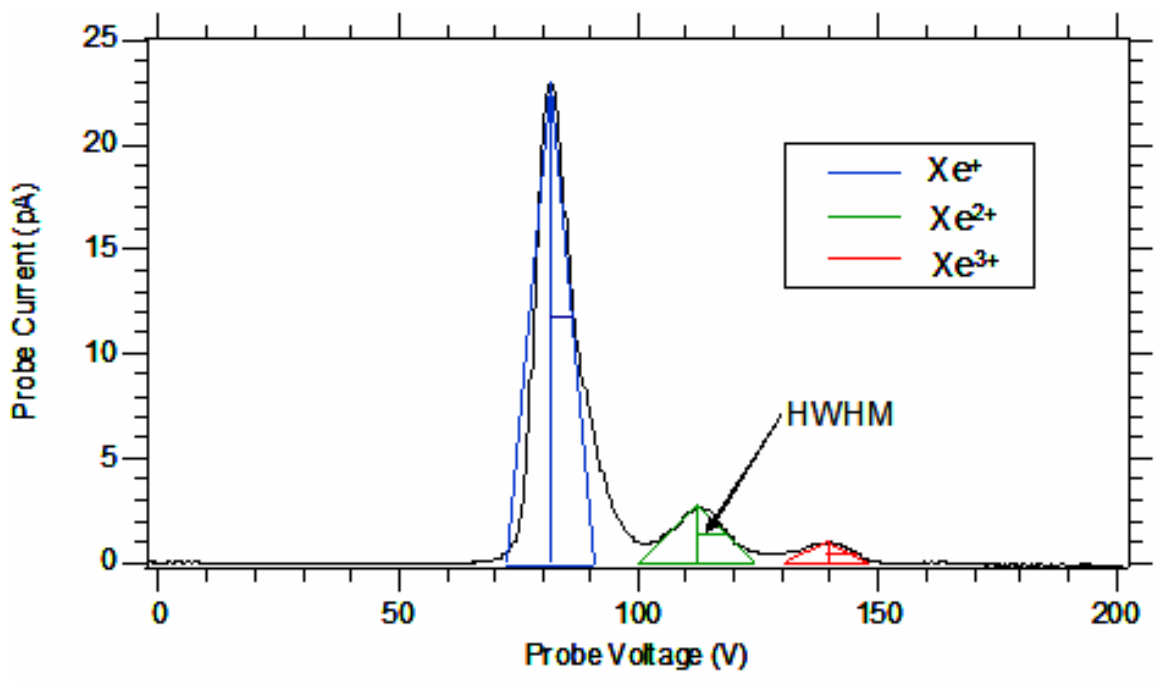

Figure 3: Illustration of the method of triangle fitting. 


\section{Method of Gaussian Fitting}

This method, suggested by Linnell [14], is an attempt to more accurately capture the total peak for each species by fitting a symmetric Gaussian profile to them. A fit is first attempted on the highest observed charge state $\left(\mathrm{Xe}^{3+}{ }^{3 n}\right.$ this investigation). The function is forced to approach zero at positive and negative infinity. Once a fit is found, the resulting function is then subtracted off the original $\mathrm{E} \times \mathrm{B}$ probe spectrum so as not to double-count current. The process is then repeated until all peaks have fits (see Figure 4). Each Gaussian profile is then integrated over all voltages to obtain the collected current for each species. As seen in Figure 4, while the Gaussian fits appear to capture more of the area under each peak than the triangle fits, it fails to fully include the overlap regions between each peak.

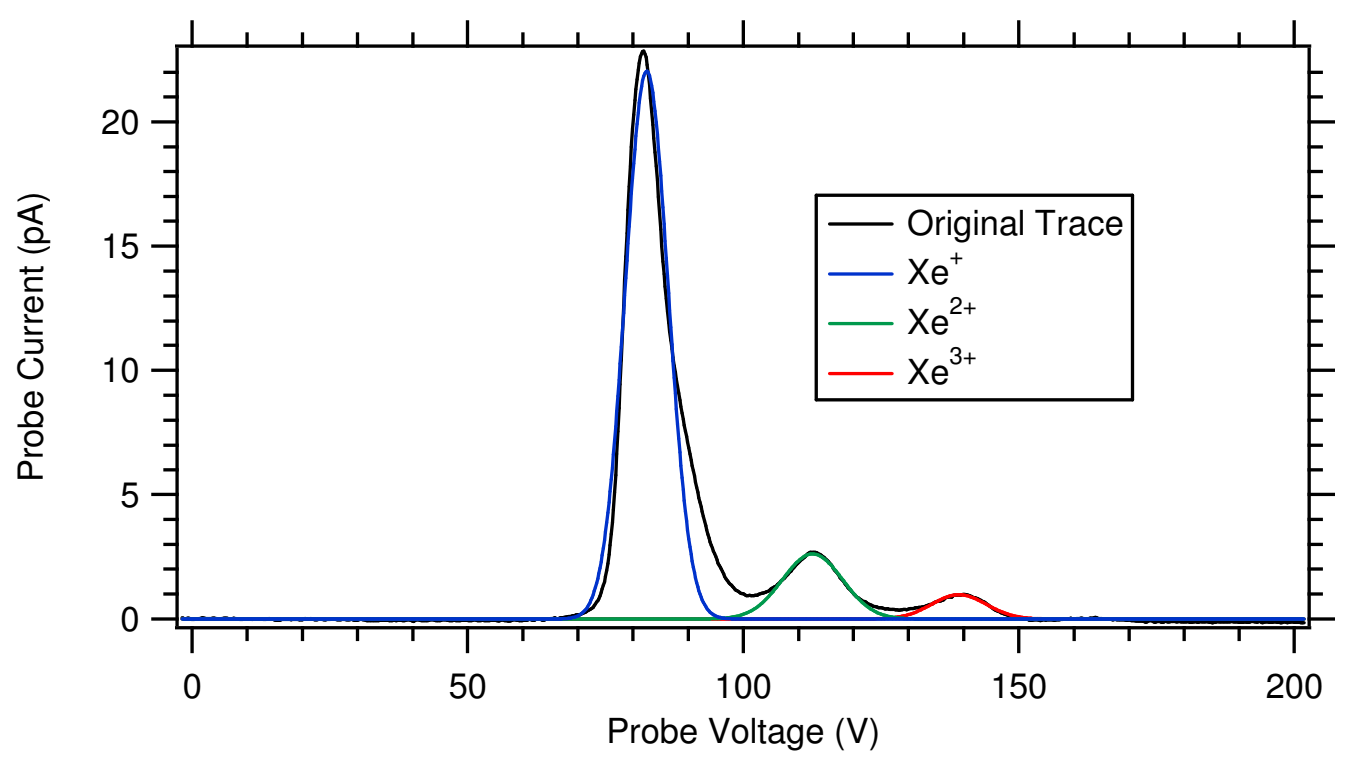

Figure 4: Illustration of the method of Gaussian fitting.

\section{Method of Variable Exponential Fitting}

This method, proposed by Kim [1], is another attempt at capturing the total peak using a functional fit. In an effort to determine a proper function to describe the spread in velocities of each species, Kim argued that the function must lie in between a Gaussian, which goes as $e^{v^{2}}$, and a Druyvesteyn profile, which goes as $e^{v^{4}}$. This is because a Gaussian function describes an equilibrium distribution due to collisional processes, while a Druyvesteyn function describes a steady-state electron or ion distribution in a uniform steady electric field with elastic collisions between particles and neutral atoms. Since the velocity distribution of ions in the plume is likely created by a combination of these two, the desired function is also likely a combination of the two functions. Thus, since the only difference between these two distributions is the value of the exponent, Kim derived a fit function based on a variable exponential model, which is shown below.

Following Kim, the current collected for a given velocity $u$ can be written as

$$
I=e \operatorname{ZnuA}_{c} .
$$

Given an energy distribution function $f(E)$, or a corresponding speed distribution function $f(C)$, we can write

$$
n \sim f(E) d E \sim f(C) d C .
$$

Assuming that the velocity of beam ions is largely one dimensional, then

$$
u \sim C \sim V_{\text {probe }} .
$$


Thus, given the variable exponential form for $f(E)$,

$$
f(E)=K^{*} E^{1 / 2} * \exp \left(-\beta *\left|\sqrt{E}-\sqrt{E_{b}}\right|^{n}\right),
$$

and that

$$
E \sim C^{2}, d E \sim C d C,
$$

one can determine the functional form for $I\left(V_{\text {probe }}\right)$,

$$
I=K^{\prime \prime} * V_{\text {probe }}{ }^{3} * \exp \left(-\beta^{\prime \prime} *\left|V_{\text {probe }}-V_{\text {probe, }, b}\right|^{n}\right),
$$

where $K$ " $\beta$ ", $V_{\text {probe, },}$, and $n$ are all fit parameters. This form is then used in the same manner as the Gaussian fit to obtain profiles for each species peak (see Figure 5). While this fit does not perfectly match each peak (in particular $\mathrm{Xe}^{+}$), it nevertheless does an excellent job capturing the overlap between each peak. This function is thus considered the most rigorous fitting method of the four investigated, at the cost of added complexity.

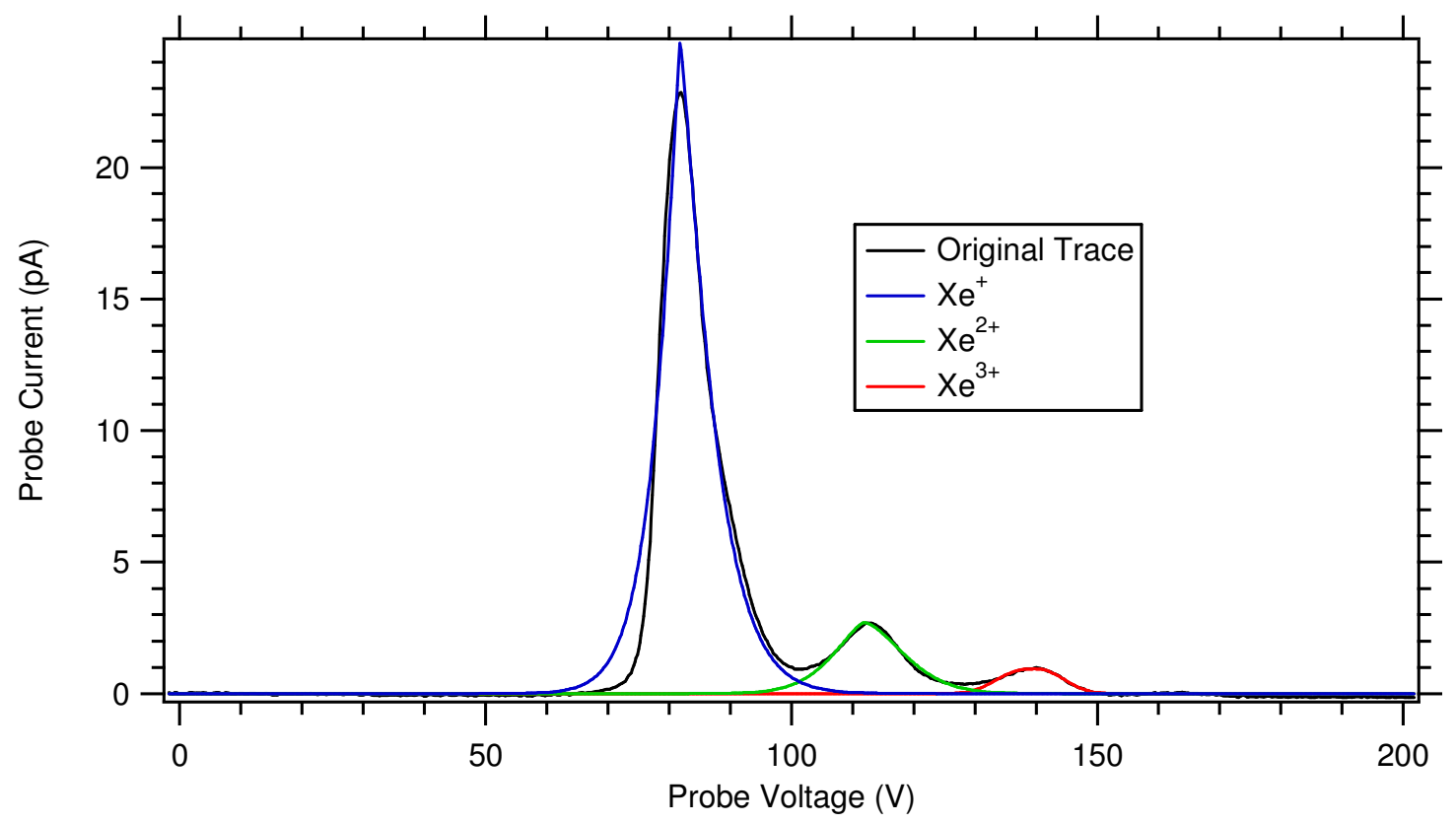

Figure 5: Illustration of the method of variable exponential fitting.

\section{Charge-Exchange Correction Methods}

In order to obtain accurate current and species fractions from an $\mathrm{E} \times \mathrm{B}$ probe, one must also consider the effects of charge exchange (CEX) collisions between beam ions and background neutrals. The presence of neutrals, either from the thruster mass flow or facility pumping limitations, can cause beam ions to become fast-moving neutrals via $\mathrm{CEX}$ collisions on their way to the $\mathrm{E} \times \mathrm{B}$ probe entrance. This causes the amount of ions to become attenuated at the probe; and since the effect of CEX collisions differs for each charge species, the relative population measured at the probe can differ significantly from the population that exits the thruster. Hall thrusters are especially sensitive to this effect compared to ions thrusters due to lower discharge voltages (and thus lower ion energies), as well as typically higher mass flow rates, which lead to larger facility backpressures (see Figure 6). Plume attenuation due to CEX loss was neglected in Ref. $[1,4,13,14]$ that may have impacted the reported ion species fractions, but will not be considered here. The method of correcting for CEX collisions on the measured charge state is discussed below. 


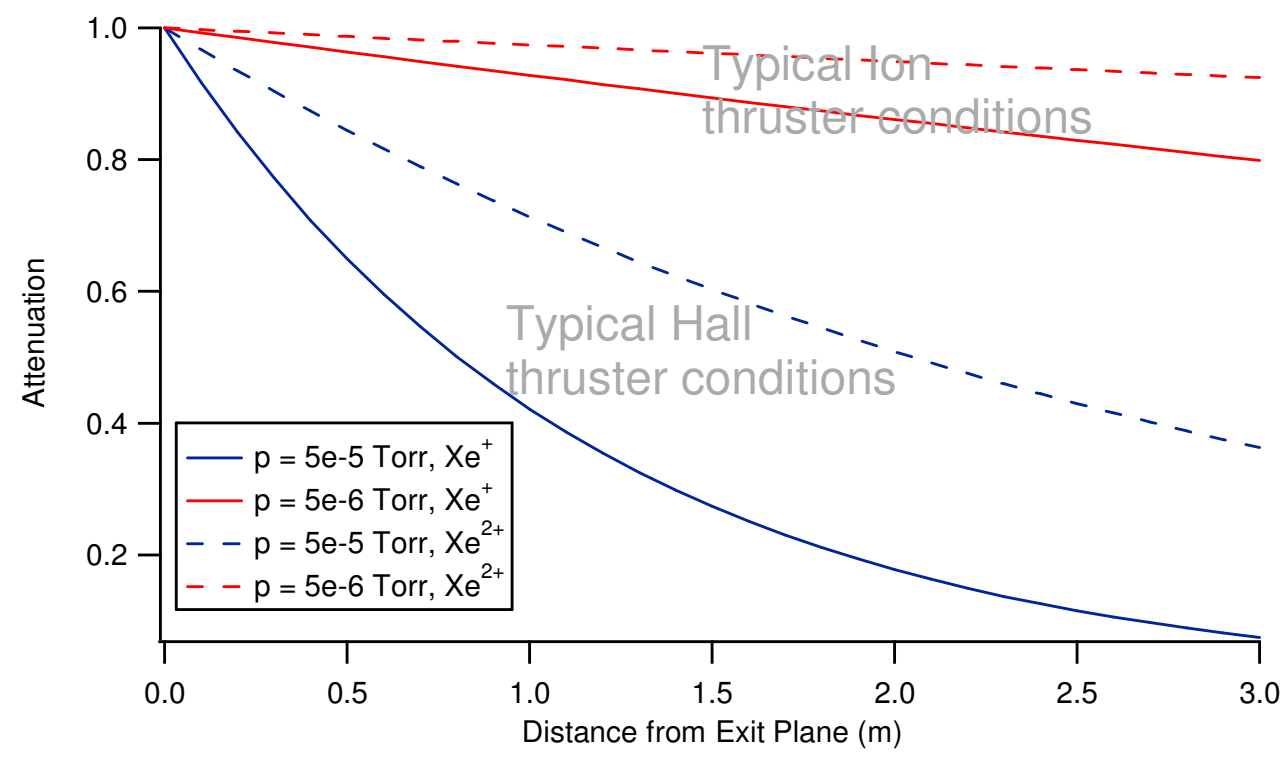

Figure 6: Comparison of beam attenuation due to charge exchange effects for singly- and doubly-ionized xenon as a function of probe distance. Note that the larger backpressures for Hall thruster operation cause a much more severe charge exchange loss.

\section{A. Baseline CEX Correction Model}

A charge exchange model for ion thrusters, derived by Anderson ${ }^{5}$, is employed and simplified in this investigation. This model assumes a one-dimensional beam consisting of ions all accelerated by the same potential, traveling through a uniform neutral background of density $n_{o}$. The relevant set of charge exchange reactions taken into account in Anderson's model are

$$
\begin{aligned}
& \left.\mathrm{Xe}^{+}+\mathrm{Xe} \rightarrow \mathrm{Xe}+\mathrm{Xe}^{+} \text {(at singly-ionized Xenon energy, cross section } \sigma_{1}\right), \\
& \mathrm{Xe}^{2+}+\mathrm{Xe} \rightarrow \mathrm{Xe}+\mathrm{Xe}^{2+}\left(\text { cross section } \sigma_{2}\right), \\
& \left.\mathrm{Xe}^{2+}+\mathrm{Xe} \rightarrow 2 \mathrm{Xe}^{+} \text {(one at doubly-ionized Xenon energy, one at thermal energy, cross section } \sigma_{3}\right), \\
& \left.\mathrm{Xe}^{+}+\mathrm{Xe} \rightarrow \mathrm{Xe}+\mathrm{Xe}^{+} \text {(at doubly-ionized Xenon energy, cross section } \sigma_{4}\right) .
\end{aligned}
$$

Equation 13-3 is regarded as an asymmetric reaction, since a new type of ion (in this case, $\mathrm{Xe}^{+}$) is created in the collision. All other reactions above are termed symmetric reactions. Cross sections are taken from Miller et al. [15], which are empirical fits to experimental data:

$$
\begin{aligned}
& \sigma_{1}, \sigma_{4}: \sigma=87.3-13.6 \log (E), \\
& \sigma_{2}=45.7-8.9 \log (E), \\
& \sigma_{3}=2
\end{aligned}
$$

where $E$ is the ion energy in $\mathrm{eV}$, and all cross sections are in $\AA^{2}\left(10^{-20} \mathrm{~m}^{2}\right)$. The third cross section varies only slightly over a wide range of energies, and thus was taken to be constant. Using the standard equations for a flux of particles traveling through a stationary background gas:

$$
\vec{\nabla} \cdot \vec{j}_{1}=-\vec{j}_{1} n_{0} \sigma_{1}
$$

\footnotetext{
${ }^{5}$ Anderson, J. "Charge-exchange collision effect on E×B probe location for NEXIS testing," Internal Memorandum, Jet Propulsion Laboratory, January 16, 2004.
} 


$$
\begin{aligned}
& \vec{\nabla} \cdot \vec{j}_{2}=-\vec{j}_{2} n_{0}\left(\sigma_{2}+\sigma_{3}\right), \\
& \vec{\nabla} \cdot \vec{j}_{3}=-\vec{j}_{3} n_{0} \sigma_{4}+\frac{\vec{j}_{2}}{2} n_{0} \sigma_{3},
\end{aligned}
$$

where $j_{1}$ is $\mathrm{Xe}^{+}$current density (at $\mathrm{Xe}^{+}$energy), $j_{2}$ is $\mathrm{Xe}^{2+}$ current density, and $j_{3}$ is $\mathrm{Xe}^{+}$current density (at $\mathrm{Xe}^{2+}$ energy). Assuming a one-dimensional beam, Anderson found:

$$
\begin{aligned}
& j_{1}=j_{10} \exp \left[-n_{0} \sigma_{1} z\right] \\
& j_{2}=j_{20} \exp \left[-n_{0}\left(\sigma_{2}+\sigma_{3}\right) z\right], \\
& j_{3}=j_{20} \frac{\sigma_{3}}{2}\left[\frac{\exp \left[-n_{0} \sigma_{4} z\right]-\exp \left[-n_{0}\left(\sigma_{2}+\sigma_{3}\right) z\right]}{\sigma_{2}+\sigma_{3}-\sigma_{4}}\right], \\
& \left(j / j_{0}\right)_{X e^{+}}=j_{1} / j_{10}, \\
& \left(j / j_{0}\right)_{X e^{2+}}=\frac{j_{2}+j_{3}}{j_{20}} .
\end{aligned}
$$

Thus, Equation 16 can be used with measured currents to determine the original current values at the thruster exit, and therefore correct for charge exchange within the plume. The background gas density can be found using a facility pressure measurement. The symmetric CEX reaction between $\mathrm{Xe}^{3+}$ and background neutrals can easily be added to this model, if it is assumed that any asymmetric reactions involving $\mathrm{Xe}^{3+}$ can be neglected:

$$
\begin{aligned}
& \left(j / j_{0}\right)_{X e^{3+}}=\exp \left[-n_{0} \sigma_{5} z\right], \\
& \sigma_{5}=16.9-3.0 \log (E) .
\end{aligned}
$$

The cross section $\sigma_{5}$ is provided by Dressler from Hanscom $\mathrm{AFB}^{6}$ and is derived from a modified Rapp-Francis CEX 1-electron model. Any calculations regarding $\mathrm{Xe}^{4+}$ have been neglected since it typically comprises less than $0.1 \%$ of the beam [1].

In the remainder of this section, a number of assumptions and simplifications to the above model are investigated and validated. These include the neglect of a higher neutral density near the thruster exit; assuming the effect of asymmetric reactions is small and thus negligible; eliminating the CEX correction for $\mathrm{Xe}^{3+}$; and assuming the acceleration voltage is equal to the discharge voltage in the CEX cross section calculations.

\section{B. Assumption of Uniform Neutral Density Field}

The model outlined in Section III-A assumes that the ion beam becomes attenuated by a neutral background gas of uniform density. While this is a reasonable assumption for the background gas caused by facility pumping limitations, the neutral density is much higher near the thruster exit due to propellant mass flow from the thruster channel. If the neutral density field $n(z)$ is assumed to be a superposition of the uniform facility density $n_{o}$ and the density field caused by the thruster neutral flux, $n_{t h}(z)$, then the attenuation fraction can be written as:

$$
\left(j / j_{0}\right)=\exp \left(-\sigma \int n_{t h}(z) d z\right) \exp \left(-\sigma n_{o} z\right)=\left(\frac{j_{1}}{j_{0}}\right)\left(\frac{j}{j_{1}}\right) .
$$

In Equation 18, $j_{1} / j_{o}$ is the attenuation fraction if there were no facility neutrals, i.e. $n_{o}=0$. Thus, $j_{1}$ would be the measured current at the probe if there were no facility effects. It can be argued that if a uniform background density

\footnotetext{
${ }^{6}$ Dressler, R. Personal Communication (Email), Hanscom AFB, October 2007.
} 
is assumed, then only the CEX with background neutrals will be corrected. Depending on the desired measurement, it may or may not be appropriate to correct for CEX with the thruster neutral flux.

In order to estimate the relative attenuation caused by thruster neutrals when compared to the attenuation by facility neutrals, two different methods are employed. The first utilizes an analytical model of neutral flow derived by $\mathrm{Katz}^{7}$. This model provides the neutral density decay as a function of distance from the thruster exit, $z$, all normalized to the value at $z=0$. This falloff is shown in Figure 7, as a two-dimensional contour plot and a onedimensional curve taken at channel centerline.
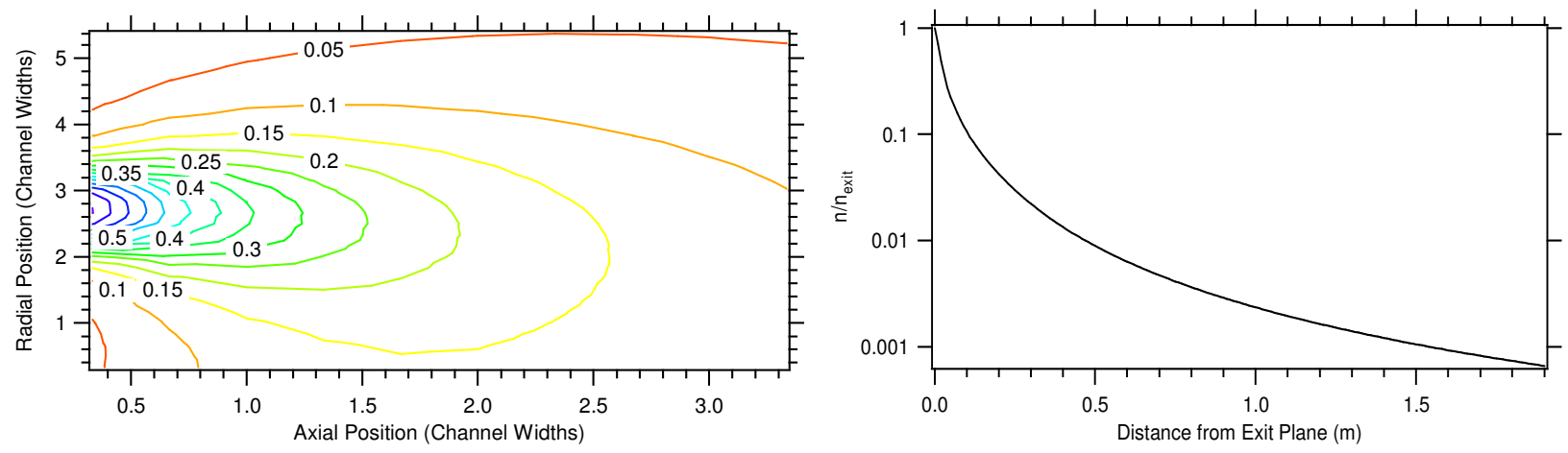

Figure 7: Calculated density decay of thruster neutrals in the plume of a Hall thruster. Left: R-Z contour plot. Right: Density decay with axial position, at channel centerline. Both plots are normalized with respect to density at $\mathrm{z}=0$.

A comparison was made using values for the operating condition at $300 \mathrm{~V}$ and $20 \mathrm{~A}$. A neutral density at thruster exit was estimated using the measured anode mass flow, an assumed mass utilization efficiency of $90 \%$ [16], and a wall temperature of $575^{\circ} \mathrm{C}$. The calculated neutral density at the thruster exit plane was found to be approximately three times larger than the measured facility background density. However, due to the rapid decay of neutral density leaving the thruster, the attenuation fraction from thruster neutrals is only 0.96 , while the fraction caused by facility neutrals is 0.60. This indicates that the CEX effects from thruster neutrals are an order of magnitude smaller than those caused by facility neutrals.

The importance of correcting for CEX with thruster neutrals was also investigated using Faraday probe traces taken at various distances from the thruster. Each of these traces were integrated over radial space and plotted as a function of $z$ as a measure of attenuation. As an estimate, the integrated currents were normalized by the discharge current for each operating condition. In order to properly compare these values to ones calculated by the CEX correction model, each species' current must be summed. It can be shown that:

$$
\frac{I(z)}{I_{o}}=\sum \Omega_{i, o} \exp \left(-\bar{n} \sigma_{i} z\right)
$$

Backpressure (or rather, "average neutral density") was iterated until a self-consistent solution was found that yielded the proper initial current fractions $\Omega_{o}$ as well as properly matched the experimental Faraday probe data. This pressure was then compared to the measured backpressure as a metric of how well the facility neutral density describes CEX attenuation (see Figure 8).

Figure 8 shows that the required backpressure to properly describe current attenuation is always higher than the measured facility pressure. The difference, however, decreases significantly as the actual amount of CEX decreases (at higher voltages and lower currents which causes lower backpressures). It should also be noted that there are sources of error with this comparison. First, all currents were normalized by discharge current, when they should have been normalized by ion beam current at the exit plane. Second, the Faraday probe traces were taken radially and thus did not likely capture the total beam current at each axial location, especially at $150 \mathrm{~V}$ and $40 \mathrm{~A}$. This

\footnotetext{
${ }^{7}$ Katz, I. Personal Communication, Jet Propulsion Laboratory, November 2007.
} 
would likely cause a larger attenuation to appear than what is caused purely by CEX, as less of the beam would be captured farther away from thruster exit. Lastly, the backpressure measurement, taken with an ionization gauge, has an inherent 20\% uncertainty. Considering these sources of error, the agreement between required and measured backpressure is quite good, and thus further evidence that CEX effects from thruster neutrals are negligible.

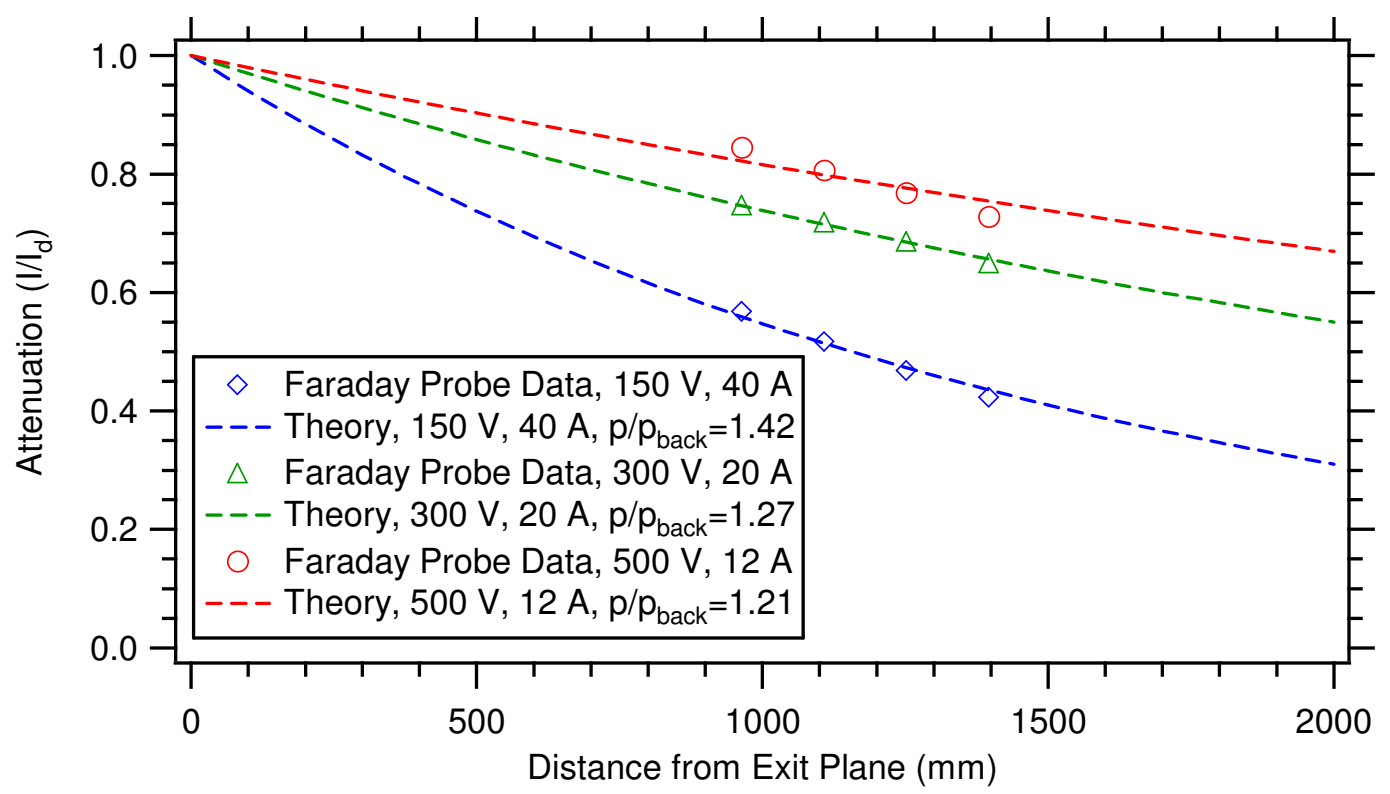

Figure 8: Comparison of Faraday probe data to theoretical values calculated using the CEX attenuation model. Note the relative agreement between the required backpressure to fit the experimental data and the measured backpressure $\left(\mathrm{p} / \mathrm{p}_{\text {back }}\right)$.

It should be noted that there may be circumstances where the effects of CEX caused by thruster neutrals are nonnegligible. For example, if a probe were placed within a few thruster diameters of the exit plane, attenuation from thruster neutrals would dominate over attenuation from facility neutrals, and thus will likely need to be accounted for. However, for ExB probes typically placed several diameters downstream of the exit plane, the amount of charge exchange caused by thruster neutrals is equivalent to that caused by facility neutrals at a backpressure of $\sim 10^{-6}$ torr, indicating that its effects can be neglected under most situations.

\section{Importance of Asymmetric Reactions}

Asymmetric reactions involve the creation of charge states different from the reactants. From Equation 14, it is evident that at moderate discharge voltages (hundreds of volts), the asymmetric cross section $\sigma_{3}$ is smaller than the symmetric cross sections by at least an order of magnitude. This indicates that the asymmetric reaction occurs far less frequently than the other CEX reactions, and yet is a source of added complexity to the CEX correction. Thus, it is worthwhile to determine the relative error in neglecting the asymmetric reaction. This is easily accomplished by setting $\sigma_{3}=0$ and comparing this simplified attenuation fraction to the complete one. Figure 9 shows the relative error in attenuation fraction for various probe distances and pressures (an acceleration voltage of $300 \mathrm{~V}$ was assumed). 


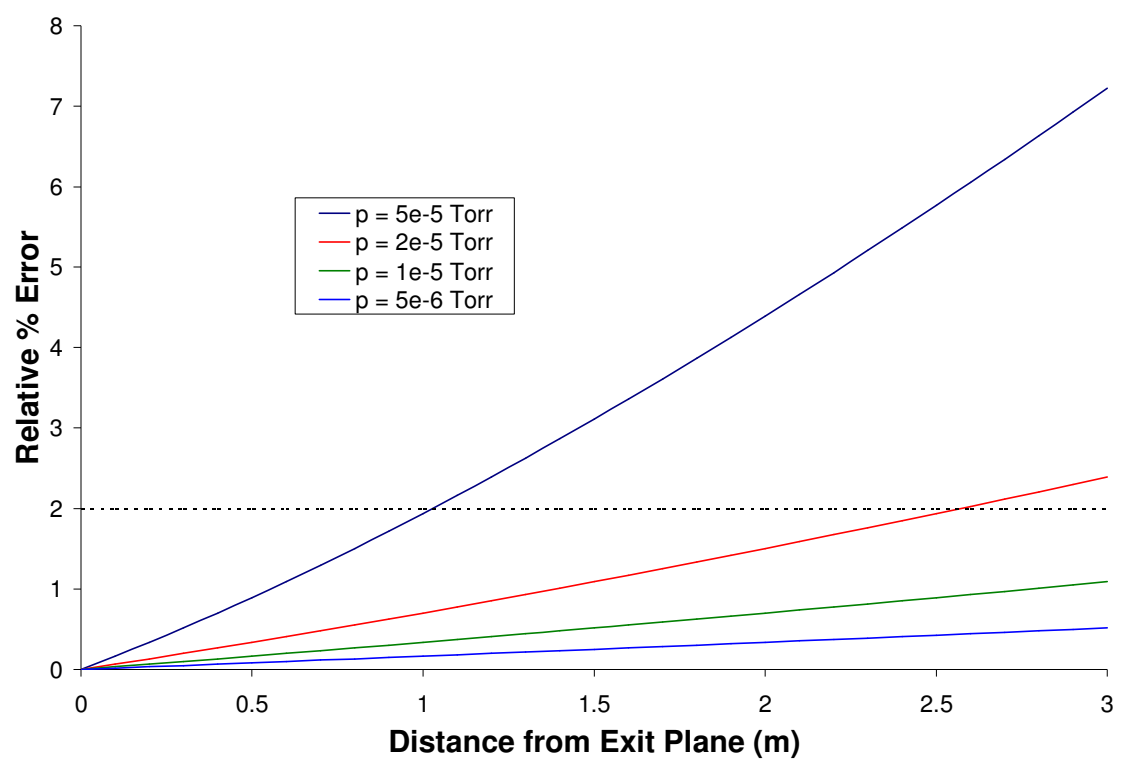

Figure 9: Relative error induced by neglecting the asymmetric reaction in correcting for charge exchange.

The error created by neglecting the asymmetric reaction is shown to be less than $2 \%$, except at large backpressures and probe distances. For typical $\mathrm{E} \times \mathrm{B}$ probe applications, probe locations are 1-2 $\mathrm{m}$ away and the operating pressure is no higher than $10^{-5}$ torr, making the error less than $1 \%$. Therefore, except under the special circumstances mentioned above, eliminating the asymmetric reaction from the CEX correction model will introduce negligible error. However, for very large backpressures and/or probes positioned very far from the thruster, asymmetric reactions should be included in the charge exchange correction.

While this in itself does not greatly reduce the complexity of the model, if we also assume that other asymmetric reactions are infrequent enough to be neglected, then the symmetric reaction of $\mathrm{Xe}^{3+}$ with background neutrals can be confidently included without the need for several additional reactions. This addition, however, has little effect on species fractions due to its small cross section. We find that across all operating conditions investigated, the relative change in $\mathrm{Xe}^{+}$and $\mathrm{Xe}^{2+}$ species fraction was less than $0.15 \%$, and the relative change in $\mathrm{Xe}^{3+}$ was less than $20 \%$. Despite this small effect, the correction for $\mathrm{Xe}^{3+}$ was left in the final CEX model for completeness.

\section{Cross Section Sensitivity to Ion Energy}

In order to calculate the CEX cross sections within the model, the relevant ion energy must be known. While this value is easily determined for ion thrusters, the acceleration voltage in Hall thrusters differs from the applied discharge voltage and must be measured, usually with a retarding potential analyzer (RPA). However, it is impractical to require the use of an RPA for every ExB probe measurement. Since the cross sections are only weakly dependent on ion energy, the error in assuming the acceleration voltage is equal to the discharge voltage was investigated. Given the general cross section formula $\sigma=a_{1}-a_{2} \log (E)$, and defining the relative error as

$$
\varepsilon=\frac{\left(j / j_{0}\right)_{V_{s}}-\left(j / j_{0}\right)_{V_{s}}}{\left(j / j_{0}\right)_{V_{s}}},
$$

one can show, assuming that $\varepsilon<<1$, that

$$
\varepsilon \approx \frac{a_{2} p z}{k T} \log \left(\frac{1}{\eta_{v}}\right)
$$


where $p$ is the backpressure, $\eta_{v}$ is the voltage utilization efficiency, $V_{d} / V_{d}$, and the rest of the symbols have their usual meaning. Figure 10 plots this error as a function of backpressure and probe distance, using the $\mathrm{Xe}^{+} \mathrm{CEX}$ cross section and assuming $\eta_{v}=0.8$ (to maximize possible error):

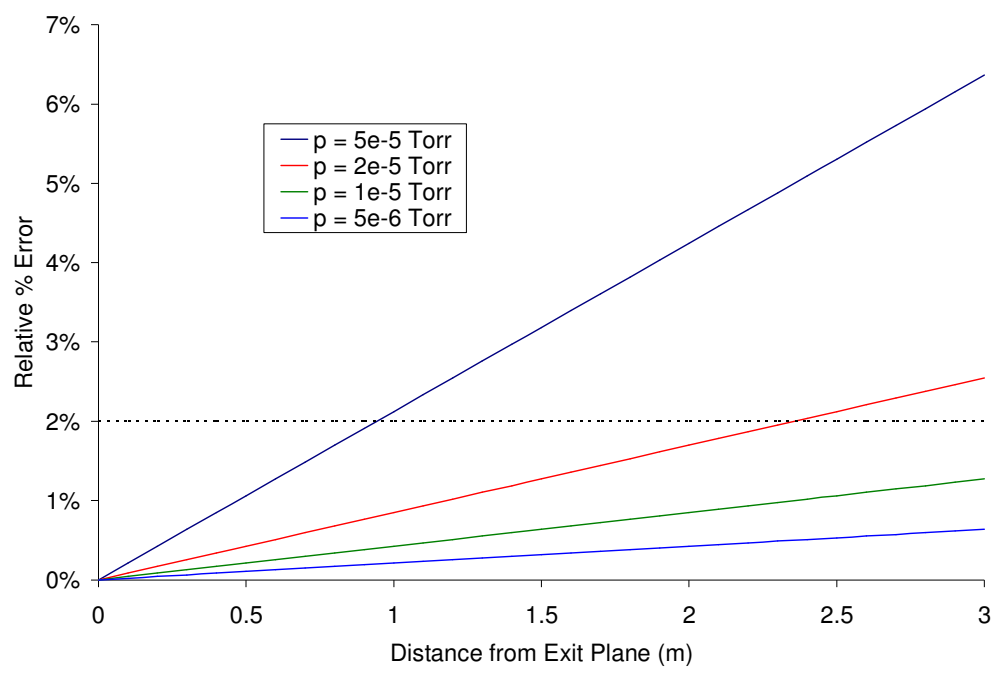

Figure 10: Error induced by assuming acceleration voltage is equal to discharge voltage in CEX cross section equations. Cross section used was symmetric $\mathrm{Xe}^{+}$reaction with background gas, with $\eta_{v}=0.8$.

For typical conditions of 1-2 m probe position and $10^{-5}$ torr, the error created is well within $1 \%$. Noting that this is likely the worst case, it is reasonable (and also practical) to use the discharge voltage to calculate the relevant ion energies in the CEX correction model.

\section{E. Simplified CEX Correction Model}

The final charge exchange correction model used in this investigation is summarized here. This model only corrects for symmetric reactions between ions and a uniform neutral background density, which is calculated using a facility pressure measurement. It also assumes an acceleration voltage equal to the discharge voltage when calculating ion energies. This allows for a simple yet reasonably accurate method of correcting for charge exchange within the plume. The final equations are

$$
\begin{array}{ll}
\left(j / j_{0}\right)_{X e^{+}}=\exp \left(-n_{0} \sigma_{1} z\right) & \sigma_{1}=87.3-13.6 \log \left(V_{d}\right), \\
\left(j / j_{0}\right)_{X e^{2+}}=\exp \left(-n_{0} \sigma_{2} z\right) & \sigma_{2}=45.7-8.9 \log \left(2 V_{d}\right), \\
\left(j / j_{0}\right)_{X e^{3+}}=\exp \left(-n_{0} \sigma_{3} z\right) & \sigma_{3}=16.9-3.0 \log \left(3 V_{d}\right) .
\end{array}
$$

\section{Results}

An ExB probe was placed on centerline $1.9 \mathrm{~m}$ downstream of a 6-kW laboratory Hall thruster, and used to measure the charge state population at eight different operating conditions (see Table 1). Each of these data sets was analyzed using the methods outlined in Sections III and IV-E. In order to more fully characterize the effects of correcting for charge exchange, three levels of correction were used: no CEX correction, correcting only the $\mathrm{Xe}^{+}$ population for CEX, which should be the most significant correction, and correcting all three species for CEX. These three levels of correction, in addition to the four methods of including species' peak width, provided twelve different methods of analysis for comparison. Due to similarity in trends across several operating conditions, only the four with the internally-mounted cathode are presented here. 


\begin{tabular}{|c|c|c|}
\hline Discharge Voltage (V) & Discharge Current (A) & Cathode Mounting Location \\
\hline 150 & 40 & Internal \\
\hline 300 & 20 & Internal \\
\hline 500 & 12 & Internal \\
\hline 300 & 10 & Internal \\
\hline 150 & 40 & External \\
\hline 300 & 20 & External \\
\hline 500 & 12 & External \\
\hline 300 & 10 & External \\
\hline
\end{tabular}

Table 1: List of Hall thruster operating conditions investigated.

Variations in species fractions, along with the relevant performance parameters, $\Sigma \Omega_{i} / Z_{i}$ and $\Sigma \Omega_{i} / N Z_{i}$ [4], were investigated across analysis methods. However, the performance parameters allow for a compact method of presenting the charge state that would otherwise have to be described with three species fractions. Furthermore, the variation in the performance parameters is of greater interest as these quantities are ultimately used in the determination of thruster efficiency. In particular, the anode efficiency for a Hall thruster can be decomposed into the product of several utilization efficiencies given as [17]

$$
\eta_{a}=\eta_{q} \eta_{v} \eta_{d} \eta_{b} \eta_{m}=\frac{\left(\sum^{\Omega_{i}} / \sqrt{Z_{i}}\right)^{2}}{\sum^{\Omega_{i}} / Z_{i}}\left(1-\frac{V_{l}}{V_{d}}\right)(\cos \theta)^{2}\left(\frac{I_{b}}{I_{d}}\right)^{2}\left(\frac{m_{X_{e}} I_{d}}{\dot{m}_{a} e}\right) \sum \frac{\Omega_{i}}{Z_{i}} .
$$

The terms directly affected by the presence of multiply-charged ions are the charge utilization efficiency and the mass utilization efficiency, given by

$$
\begin{gathered}
\eta_{q}=\frac{\left(\sum \Omega_{i} / \sqrt{Z_{i}}\right)^{2}}{\sum^{\Omega_{i}} / Z_{i}}, \\
\eta_{m}=\left(\frac{m_{X_{e}} I_{d}}{\dot{m}_{a} e}\right)\left(\frac{I_{b}}{I_{d}}\right) \sum \frac{\Omega_{i}}{Z_{i}}=\left(\frac{m_{X e} I_{d}}{\dot{m}_{a} e}\right)\left(\frac{I_{b}}{I_{d}}\right) \alpha_{m} .
\end{gathered}
$$

Therefore, variations in $\eta_{q}$ and $\alpha_{m}$ are considered to have a direct correlation with variations in the calculated thruster efficiency. Since it was found that $\alpha_{m}$ varied more significantly than $\eta_{q}$ over all test cases, only variations in $\alpha_{m}$ are presented here. The effective charge state $Q$, defined as $\sum \zeta_{i} Z_{i}$, is another relevant parameter when discussing the charge state population [18]; however, since it has no direct impact on the calculation of efficiency in the above model, it is not presented in detail here. See Appendix A for a list of calculated current and species fractions, as well as $\alpha_{m}, \eta_{q}$, and $Q$ for all operating conditions investigated.

The purpose of such a comparison across analysis methods is to quantify the importance of correcting for peak width and charge exchange in determining current and species fractions, and thus propose a method that yields the best balance of accuracy and simplicity. Since the most complex method, and arguably the most accurate, is the variable exponential fit with full CEX correction, all methods are compared relative to this one. Thus, all results will be presented in terms of relative percent difference between $\alpha_{m}$ for each method and $\alpha_{m}$ for the most complex method. Results across four operating conditions are presented below. 


\section{A. Operating Condition of $150 \mathrm{~V}, 40 \mathrm{~A}$}

This particular condition of low voltage and high current represents a case of potentially large amounts of charge exchange collisions within the plume. Facility pressure was measured to be $2.6 \times 10^{-5}$ torr, corrected for xenon. The variation in $\alpha_{m}$ with each analysis method is shown in Figure 11.

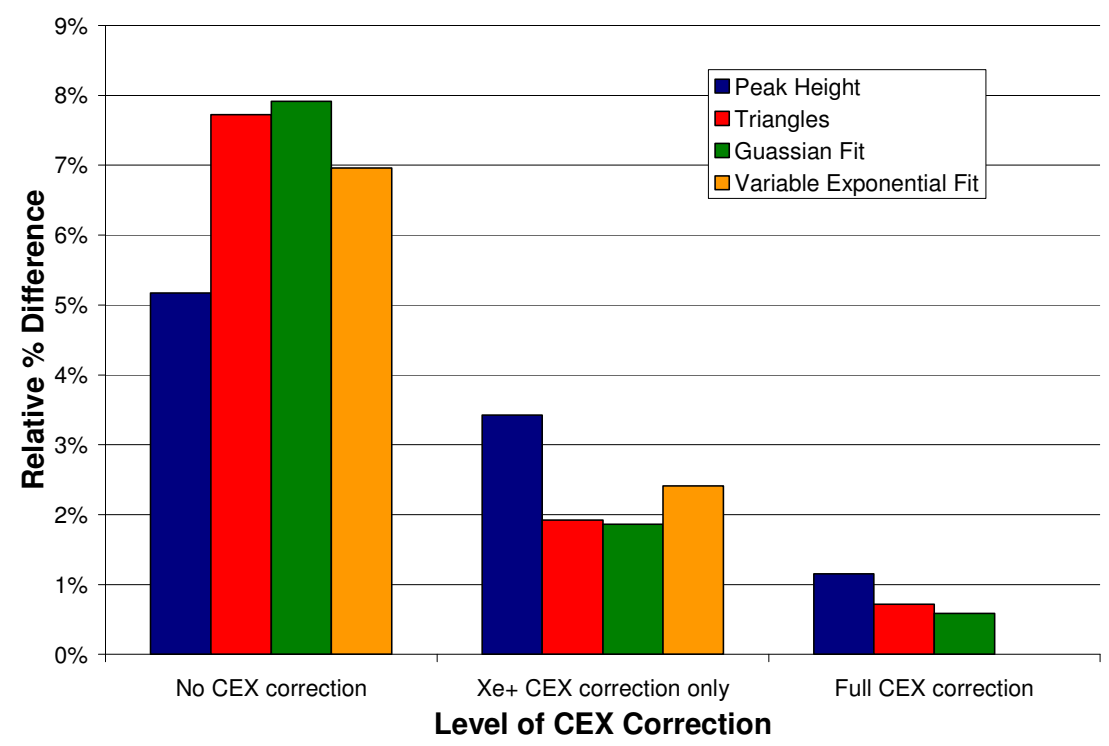

Figure 11: Comparison of $\alpha_{m}$ between several analysis methods at $150 \mathrm{~V}, 40 \mathrm{~A}$, relative to the full CEX correction using the variable exponential fit. Notice the dominance of the effects of CEX correction over the differences in peak width methods.

From Figure 11 it is evident that the effects of correcting for charge exchange dominate over the effects of peak width, as expected. Relative errors reached as high as almost $8 \%$, which is extremely significant in the context of efficiency analysis. Once the full CEX correction is applied, the relative error drops to nearly within $1 \%$, regardless of which method is used to include peak width. It is interesting to note that the error induced from correcting for peak width but not CEX is larger than correcting for nothing at all. This is a characteristic that will be shown at other operating conditions as well.

\section{B. Operating Condition of $300 \mathrm{~V}, 20 \mathrm{~A}$}

This particular condition of moderate voltage and current represents a more typical case of Hall thruster operation. Facility pressure was measured to be $1.6 \times 10^{-5}$ torr, corrected for xenon. The variation in $\alpha_{m}$ with each analysis method is shown in Figure 12. 


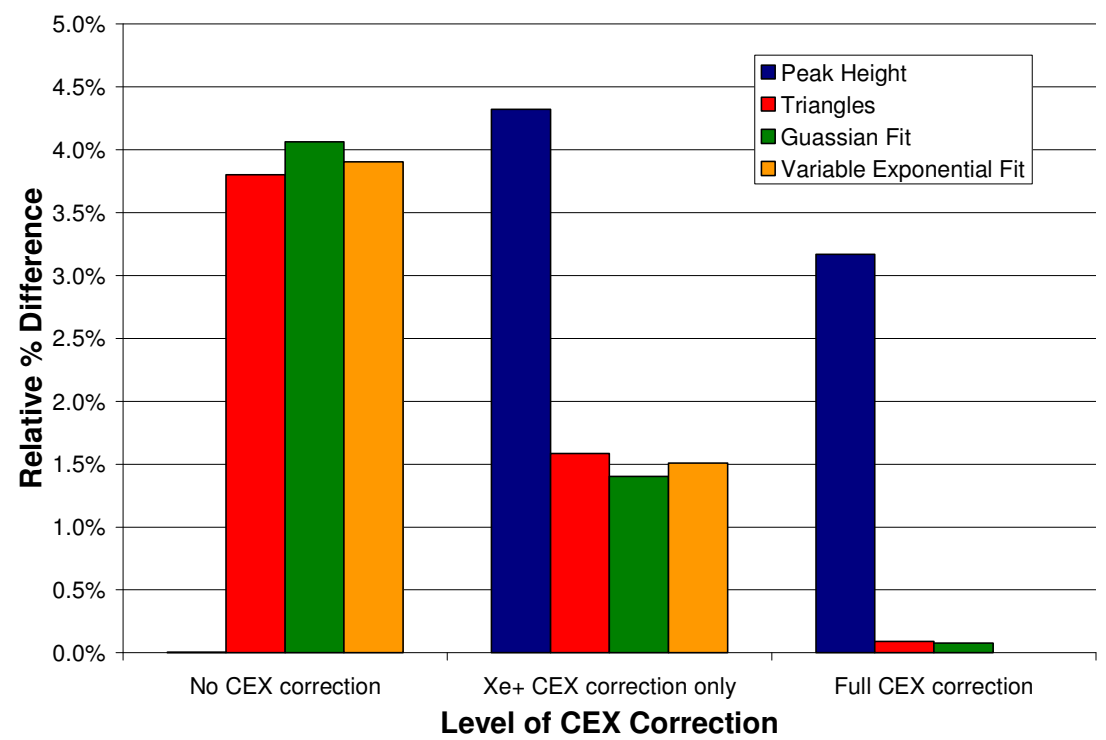

Figure 12: Comparison of $\alpha_{m}$ between several analysis methods at $300 \mathrm{~V}, 20 \mathrm{~A}$, relative to the full CEX correction using the variable exponential fit. Notice that the largest errors occur from correcting for either CEX or peak width.

The most noticeable feature at this operating condition is that the relative error is maximized when either peak width OR charge exchange collisions are corrected for. As with the data at $150 \mathrm{~V}, 40 \mathrm{~A}$, there is negligible difference between any method that takes peak width into account. Relative difference is minimized to within $0.1 \%$ using a full CEX correction and any method including peak width. It is interesting to note that at this particular operating condition, using peak heights and not correcting for CEX gave a nearly identical result to the variable exponential fit with full CEX correction method. This can be explained by noting that the two correction factors have opposite effects on the calculated charge state. As peaks tend to be wider for higher charge states, including them shifts the overall charge state to a larger value. Conversely, $\mathrm{CEX}$ affects $\mathrm{Xe}^{+}$more than higher charge states, so correcting for this shifts the charge state closer to 1 . These two competing effects give the interesting result that correcting for either peak width or CEX will typically yield a higher difference than correcting for nothing at all using the simplest method.

\section{Operating Condition at $500 \mathrm{~V}, 12 \mathrm{~A}$}

The particular condition of higher voltage and lower current is a case where a much smaller amount of CEX likely occurs within the plume. Facility pressure was measured to be $1.2 \times 10^{-5}$ torr, corrected for xenon. The variation in $\alpha_{m}$ with each analysis method is shown in Figure 13. 


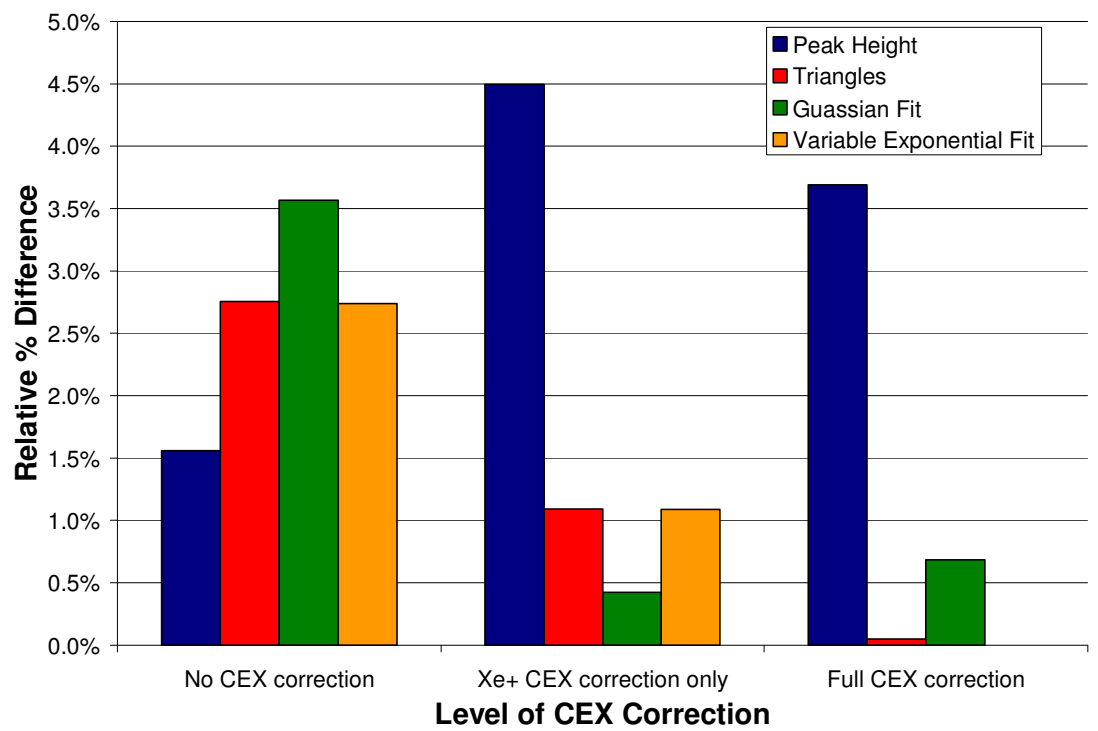

Figure 13: Comparison of $\alpha_{m}$ between several analysis methods at $500 \mathrm{~V}, 12 \mathrm{~A}$, relative to the full CEX correction using the variable exponential fit.

As with $300 \mathrm{~V}$ and $20 \mathrm{~A}$, the largest differences occur when either peak width or CEX is corrected for but not the other. In order for the relative difference to be within 1\%, only a full CEX correction along with any inclusion of peak width is sufficient. This trend is present in all operating conditions investigated.

\section{Operating Condition at $300 \mathrm{~V}, 10 \mathrm{~A}$}

This condition was taken at a discharge power that is half of the power of the other test cases. The discharge current is also the lowest investigated, with a facility pressure measured to be $9.7 \times 10^{-6}$ torr, corrected for xenon. The variation in $\alpha_{m}$ with each analysis method is shown in Figure 14.

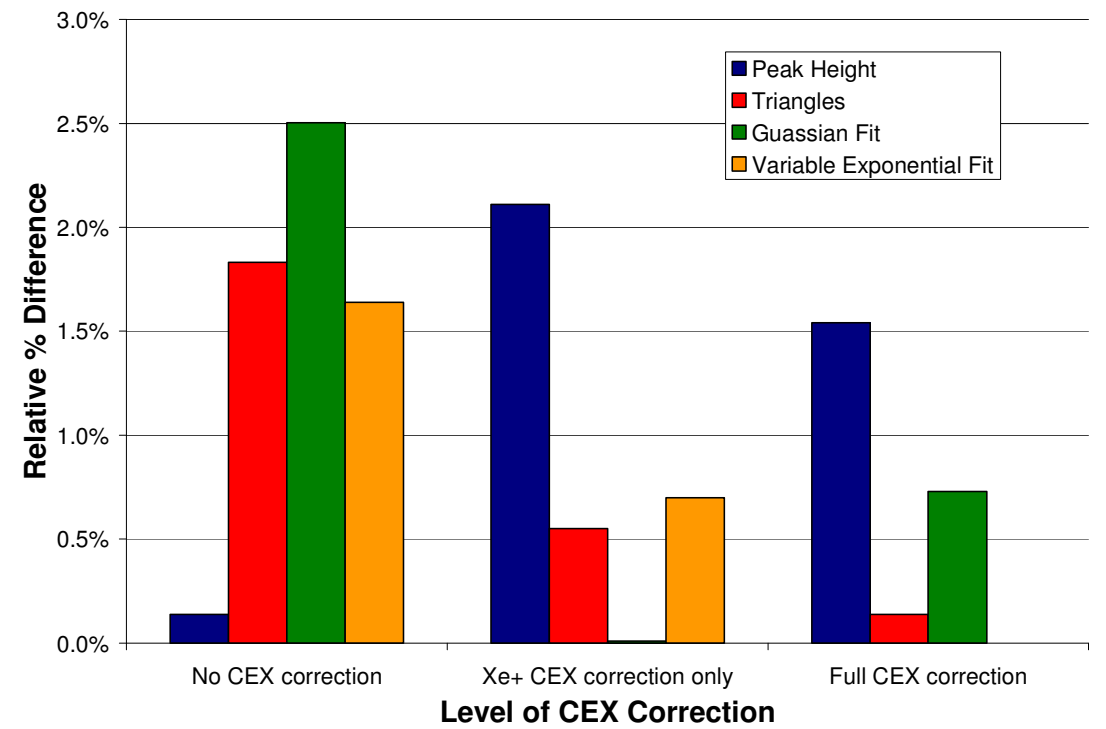

Figure 14: Comparison of $\alpha_{m}$ between several analysis methods at $300 \mathrm{~V}, 10 \mathrm{~A}$, relative to the full CEX correction using the variable exponential fit.

This particular case shows the lowest relative differences calculated, with a maximum of only $2.5 \%$. Once again, the maximum differences occur when either peak widths or CEX are accounted for. At this particular pressure, it appears that only a correction to $\mathrm{Xe}^{+}$with an inclusion of peak width is required to be within $1 \%$ of the variable 
exponential fit and full CEX correction method. This indicates that the correction of charge exchange for higher charge states is less significant at lower pressures, which is to be expected.

\section{Discussion}

Based on the results from the eight different operating conditions investigated, it is recommended that the method of triangle fitting with the full CEX correction be used to analyze ExB probe spectra from Hall thruster plumes. Results from this method are consistently within $1 \%$ of the results from the most complex method used over a wide range of operating conditions. It has been shown that correcting for charge exchange can yield a significantly different charge state, especially at high facility pressures, while the triangle fitting method provides effectively the same results when compared to higher order fit methods. Thus, this method provides the best balance between simplicity and accuracy over all operating conditions investigated. It should be noted that the method of triangle fitting is sufficient merely in calculating current and species fractions; this approximation does not capture much of the detail of each species peak, and therefore should not be used for analyzing the physics of the VDF.

In order to prevent facility effects from excessively altering the ExB probe measurement, it is recommended that the attenuation fraction of $\mathrm{Xe}^{+}$be kept above 0.7 . While this is an arbitrary choice, it provides a reasonable guideline for controlling the magnitude of charge exchange effects within the plume. Since the CEX cross section of $\mathrm{Xe}^{+}$with neutrals is only weakly dependent on ion energy, this attenuation fraction guideline can be roughly converted into a " $p z$ " factor of backpressure multiplied by probe distance from the exit plane, which is more practical. Thus, in order to keep facility effects at a reasonable level, it is recommended that $p z \leq 2$, where $p$ is the facility backpressure in units of $10^{-5}$ torr, and $z$ is the distance from the thruster exit plane to the $\mathrm{E} \times \mathrm{B}$ probe in meters. In this study, $p z$ varied between 1.8 at $10 \mathrm{~A}$ and 4.9 at $40 \mathrm{~A}$. While the above recommendation was not strictly met, $p z$ was still kept at a reasonable level for all operating conditions except $40 \mathrm{~A}$, and therefore the results presented above are considered valid. A study performed by Reid et al. [9] utilized the above methods on ExB probe spectra taken at various distances from the thruster. After correction, excellent results were obtained that were consistent across all distances tested, providing further validation of the recommended analysis method.

\section{A. Error Analysis}

Each analysis method investigated above provides a certain amount of additional uncertainty in the calculated charge state, due to the larger amount of data processing required. Major sources of uncertainty include the facility pressure measurement, estimated at $20 \%$, the cross sections within the charge exchange correction model, estimated at $20 \%$ for $\mathrm{Xe}^{+}$and $30 \%$ for $\mathrm{Xe}^{2+}$ and $\mathrm{Xe}^{3+}$, and each fitting method. Taking into account uncertainties in pressure and cross sections, the charge exchange correction generates an additional relative uncertainty of up to $2-8 \%$ in $\mathrm{Xe}^{+}$ current fraction, $15-50 \%$ in $\mathrm{Xe}^{2+}$ current fraction, and $10-30 \%$ in $\mathrm{Xe}^{3+}$ current fraction. This corresponds to an additional relative uncertainty of up to $1-4 \%$ for $\alpha_{m}$. It should be noted that the quantities calculated without the charge exchange correction always lay outside these uncertainty bounds, indicating that the correction is still certainly worth doing. Also, the amount of uncertainty is directly related to the facility pressure, and consequently the amount of charge exchange within the plume. Therefore, a lower backpressure will result in smaller uncertainty bounds created by the charge exchange correction.

Errors induced by using the triangle fitting method can be estimated by comparing calculated values between this method and the more rigorous variable exponential fit method. The relative amount of error was found to be up to $2.5 \%$ for $\mathrm{Xe}^{+}$current fraction, $20 \%$ for $\mathrm{Xe}^{2+}$ current fraction, and $25 \%$ for $\mathrm{Xe}^{3+}$ current fraction, corresponding to errors of up to $1 \%$ for $\alpha_{m}$. Noting that all of above estimates are maximum deviations, the combined standard uncertainty has been estimated as $3 \%$ in $\mathrm{Xe}^{+}, 20 \%$ in $\mathrm{Xe}^{2+}$ and $\mathrm{Xe}^{3+}$, and $2 \%$ in $\alpha_{m}$.

\section{B. Species Fraction Spatial Dependence}

While the above described methods are applicable to an $\mathrm{E} \times \mathrm{B}$ probe spectrum taken anywhere in the plume, measurements are typically taken along thruster centerline 1-2 m downstream of the exit plane. In order to characterize the species population within the plume, one must consider any spatial variation which occurs and its effect on the overall charge state. Experiments performed by Kim [1] and simulations performed by Katz [19] on the SPT-100 have shown that species fractions can vary significantly off-centerline, showing that there is a larger population of multiply-charged species farther off axis (see Figure 15). This indicates that a single-point centerline measurement may not be sufficient to describe accurately the average charge state of ions exiting the thruster. 


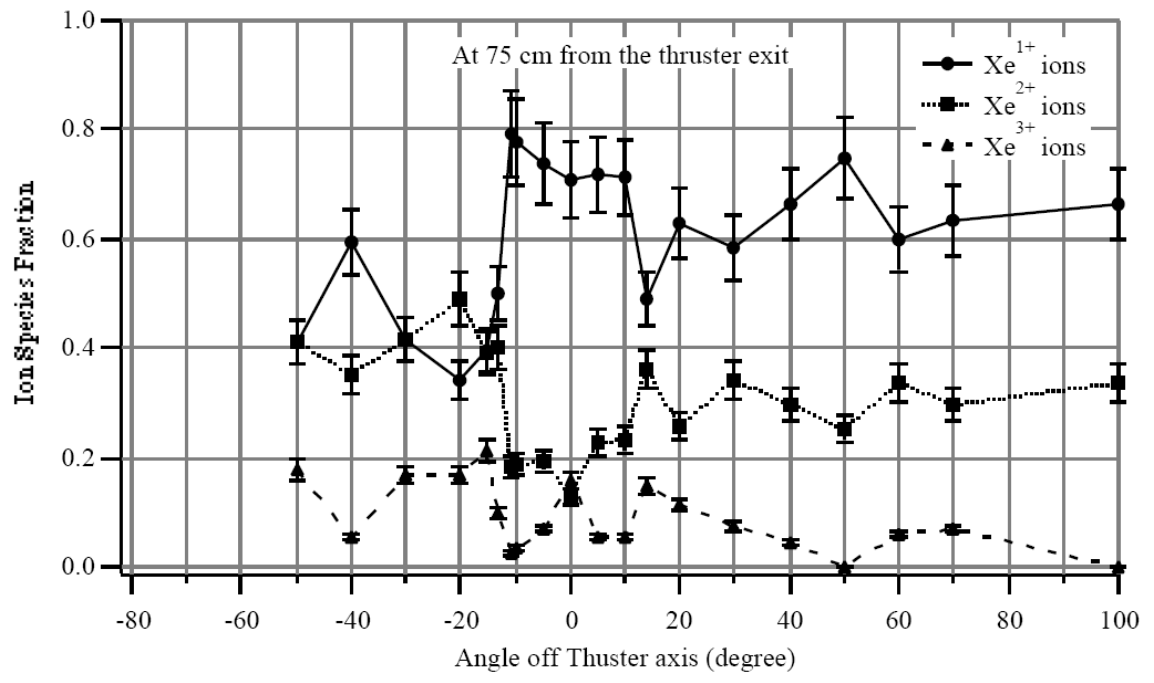

Figure 15: Species fraction variation with angle for an SPT-100 taken $75 \mathrm{~cm}$ from thruster exit. Beyond $10^{\circ}$ there is a noticeable increase in multiply-charged species. Figure courtesy of Kim [1].

Experiments performed by Reid et al. [9] on a $6 \mathrm{~kW}$ laboratory Hall thruster verifies the existence of higher charge states off thruster centerline. An average $\alpha_{m}$ was found over the spatial domain tested, weighted by the local current density, and compared to the values yielded by the thruster centerline and channel centerline measurements (see Figure 16). This average varied up to $3.5 \%$ from the single-point thruster centerline measurement, but only by $1.5 \%$ when compared to the single-point channel centerline measurement. It was thus suggested that a measurement taken at channel centerline would be sufficient for studies concerned with thruster behavior over large throttling ranges, with appropriate error bounds to accommodate the spatial variation within the plume. However, for studies focused on fine changes in thruster performance, the resulting error in a single-point measurement may not be tolerable and determining plume-averaged quantities will be necessary.

\section{Conclusion}

Various analysis methods were applied to $\mathrm{E} \times \mathrm{B}$ probe spectra taken in the plume of a $6-\mathrm{kW}$ laboratory Hall thruster over a wide range of operating conditions. These methods incorporated varying degrees of correction for the width of species peaks as well as charge exchange collision effects within the plume, in order the determine the importance of these effects on the calculated species fractions and efficiency terms. It has been shown that the use of a simple model using triangles to approximate the velocity distribution function for each species peak is sufficient to capture the effect of peak width on calculated species fraction. While the extent of charge exchange varies strongly with the facility backpressure, it has been found that the effects of charge exchange are extremely significant at pressures larger than $10^{-5}$ torr, and thus should be corrected for. However, a simplified method of correction has been suggested that can easily be implemented and generate small uncertainty over the majority of 
cases. Most importantly, it has been shown that the amount of charge exchange caused by the neutral thruster flux (as opposed to facility neutrals) is negligible except when the probe is very close to the exit plane (a few thruster diameters). In order to keep the amount of charge exchange within reasonable limits, it is recommended that the product of $p$ and $z$ be kept below 2, where $p$ is the facility pressure in units of $10^{-5}$ torr, and $z$ is the distance from thruster exit to the probe. Lastly, it has been suggested by Reid et al. [9] that a single-point measurement at thruster centerline is insufficient to properly characterize the charge state of the thruster plume. However, a single-point measurement taken at channel centerline can minimize the error to within $1.5 \%$ when compared to the average of a more complete spatial map. The methods suggested in this paper should allow for a more accurate determination of the charge state of Hall thruster plumes, and thus facilitate a more accurate understanding of the competing processes affecting overall thruster efficiency.

\section{Appendix A - Selected Tabulated Values Across Operating Conditions}

Below is a list of the current fractions, species fractions, mass utilization correction factors for multiply-charged ions, charge utilization efficiencies, and effective charge states for the operating conditions investigated in this study. In the table, INT corresponds to an internally-mounted cathode, while EXT corresponds to an externallymounted cathode. The results below were calculated using the recommended method of triangle fitting with full CEX corrections. It should be noted that with the exception of $150 \mathrm{~V}, 40 \mathrm{~A}$ with an externally-mounted cathode, values are nearly identical across operating conditions at the same discharge power.

\begin{tabular}{|c|c|c|c|c|c|c|c|c|c|}
\hline & $\Omega\left(\mathrm{Xe}^{+}\right)$ & $\Omega\left(\mathrm{Xe}^{2+}\right)$ & $\Omega\left(\mathrm{Xe}^{3+}\right)$ & $\zeta\left(\mathrm{Xe}^{+}\right)$ & $\zeta\left(\mathrm{Xe}^{2+}\right)$ & $\zeta\left(\mathrm{Xe}^{3+}\right)$ & $\alpha_{m}$ & $\eta_{q}$ & Q \\
\hline $150 \mathrm{~V}, 40 \mathrm{~A}, \mathrm{INT}$ & 0.800 & 0.154 & 0.046 & 0.927 & 0.063 & 0.010 & 0.892 & 0.981 & 1.08 \\
\hline $150 \mathrm{~V}, 40 \mathrm{~A}, \mathrm{EXT}$ & 0.845 & 0.123 & 0.032 & 0.945 & 0.049 & 0.006 & 0.917 & 0.985 & 1.06 \\
\hline $300 \mathrm{~V}, 10 \mathrm{~A}, \mathrm{INT}$ & 0.858 & 0.106 & 0.036 & 0.951 & 0.042 & 0.007 & 0.923 & 0.985 & 1.06 \\
\hline $300 \mathrm{~V}, 10 \mathrm{~A}, \mathrm{EXT}$ & 0.869 & 0.098 & 0.033 & 0.955 & 0.038 & 0.007 & 0.929 & 0.987 & 1.05 \\
\hline $300 \mathrm{~V}, 20 \mathrm{~A}, \mathrm{INT}$ & 0.802 & 0.148 & 0.050 & 0.928 & 0.060 & 0.012 & 0.893 & 0.980 & 1.08 \\
\hline $300 \mathrm{~V}, 20 \mathrm{~A}, \mathrm{EXT}$ & 0.798 & 0.160 & 0.042 & 0.925 & 0.065 & 0.010 & 0.892 & 0.981 & 1.08 \\
\hline $500 \mathrm{~V}, 12 \mathrm{~A}, \mathrm{INT}$ & 0.798 & 0.147 & 0.055 & 0.927 & 0.060 & 0.013 & 0.890 & 0.980 & 1.08 \\
\hline $500 \mathrm{~V}, 12$ A, EXT & 0.790 & 0.155 & 0.055 & 0.924 & 0.064 & 0.012 & 0.886 & 0.979 & 1.09 \\
\hline
\end{tabular}

Acknowledgments

The authors would like to thank John Anderson and Ira Katz at JPL for their assistance on formulation and development of the charge exchange correction model used in this paper, along with Rainer Dressler at Hanscom AFB for providing additional xenon charge exchange cross sections.

The research described in this paper was carried out at the Jet Propulsion Laboratory, California Institute of Technology, under a contract with the National Aeronautics and Space Administration. 


\section{References}

[1] Kim, S.-W., "Experimental Investigations of Plasma Parameters and Species-Dependent Ion Energy Distribution in the Plasma Exhaust Plume of a Hall Thruster," Ph.D. Dissertation, Aerospace Engineering, University of Michigan, 1999.

[2] Kim, S.-W. and Gallimore, A. D., "Plume study of a 1.35-kW SPT-100 using an ExB probe," Journal of Spacecraft and Rockets 39, 6 (2002).

[3] King, L. B. and Gallimore, A. D., "Mass Spectral Measurements in the Plume of an SPT-100 Hall Thruster," Journal of Propulsion and Power 16, 6, 1086-1092 (2000).

[4] Hofer, R. R., "Development and Characterization of High-Efficiency, High-Specific Impulse Xenon Hall Thrusters," Ph.D Dissertation, Aerospace Engineering, The University of Michigan, 2004.

[5] Hofer, R. R. and Gallimore, A. D., "High-Specific Impulse Hall Thrusters, Part 2: Efficiency Analysis," Journal of Propulsion and Power 22, 4, 732-740 (2006).

[6] Leal-Quiros, E. and Prelas, M. A., "New tilted-poles Wien filter with enhanced performance," Review of Scientific Instruments 60, 3 (1989).

[7] Quiros, E. L., "Some experimental facts that indicate the elimination of astigmatism in ion beams with separators using cross electric and magnetic fields," Journal of Applied Physics 52, 3 (1981).

[8] Seliger, R. L., "ExB Mass-Separator Design," Journal of Applied Physics 43, 5, 2352-2357 (1972).

[9] Reid, B. M., Shastry, R., Gallimore, A. D., and Hofer, R. R., "Angularly-Resolved ExB Probe Spectra in the Plume of a 6-kW Hall Thruster," Presented at the 44th AIAA/ASME/SAE/ASEE Joint Propulsion Conference, AIAA-2008-5287, Hartford, CT, July 20-23, 2008.

[10] Anderson, J. R. and Fitzgerald, D., "Fullerene propellant research for electric propulsion," Presented at the 32nd AIAA/ASME/SAE/ASEE Joint Propulsion Conference and Exhibit, AIAA-1996-3211, Lake Buena Vista, FL, Jul. 1-3, 1996.

[11] Sengupta, A., Brophy, J. R., Anderson, J. R., Garner, C., Banks, B. et al., "An Overview of the Results from the 30,000 Hr Life Test of Deep Space 1 Flight Spare Ion Engine," Presented at the 40th AIAA/ASME/SAE/ASEE Joint Propulsion Conference and Exhibit, AIAA-2004-3608, Fort Lauderdale, FL, Jul. 11-14, 2004.

[12] Randolph, T. M. and Polk, J. E., "An overview of the nuclear electric xenon ion system (NEXIS) activity," Presented at the Space 2004 Conference and Exhibit, AIAA-2004-5909, San Diego, CA, Sept. 28-30, 2004.

[13] Beal, B. E., "Clustering of Hall-effect Thrusters for High-Power Electric Propulsion Applications," Ph.D Dissertation, Aerospace Engineering, The University of Michigan, 2004.

[14] Linnell, J. A., "An Evaluation of Krypton Propellant in Hall Thrusters," Ph.D. Dissertation, Aerospace Engineering, University of Michigan, 2007.

[15] Miller, J. S., Pullins, S. H., Levandier, D. J., Chiu, Y.-H., and Dressler, R. A., "Xenon charge exchange cross sections for electrostatic thruster models," Journal of Applied Physics 91, 3, 984-991 (2002).

[16] Jameson, K. K., Goebel, D. M., Hofer, R. R., and Watkins, R. M., "Cathode Couplings in Hall Thrusters," Presented at the 30th International Electric Propulsion Conference, IEPC-2007-278, Florence, Italy, September 17-20, 2007.

[17] Hofer, R. R., Katz, I., Mikellides, I. G., Goebel, D. M., Jameson, K. K. et al., "Efficacy of Electron Mobility Models in Hybrid-PIC Hall Thruster Simulations," Presented at the 44th AIAA/ASME/SAE/ASEE Joint Propulsion Conference, AIAA-2008-4924, Hartford, CT, Jul. 20-23, 2008.

[18] Larson, C. W., Brown, D. L., and Hargus, W. A., "Thrust Efficiency, Energy Efficiency, and the Role of VDF in Hall Thruster Performance Analysis," Presented at the 43rd AIAA/ASME/SAE/ASEE Joint Propulsion Conference, AIAA2007-5270, Cincinnati, OH, Jul. 8-11, 2007. 
[19] Katz, I., Hofer, R. R., and Goebel, D. M., "Ion Current in Hall Thrusters," IEEE Transactions on Plasma Science (accepted for publication, 2008). 